\title{
Tratamento gravado em vídeo diretamente observado: uma ferramenta possível em saúde mental
}

\author{
Rodrigo Mauro Lacerda Azevedo* \\ Rodrigo de Santana Geraldo Braga** \\ Helena Moraes Cortes*** \\ Paula Hayasi Pinho****
}

\section{Resumo}

Descreve-se a experiencia da utilização do tratamento gravado em vídeo um serviço público de saúde mental, em uma cidade do Recôncavo da Bahia. Os vídeos foram enviados por Serviço de Mensagens Multimídia (SMS). Tal técnica foi decisiva, para que uma usuária do serviço de saúde mental fizesse uso diariamente, de suas medicações sem qualquer falha, o que possibilitou uma rápida melhora de sua produção delirante. Sugere-se que a introdução das gravações diretamente observadas no período agudo dos quadros de transtornos mentais graves poderia favorecer um melhor desfecho terapêutico, pois pode trazer a segurança do correto uso das medicações nestes momentos.

Palavras-chave: Saúde mental. Adesão à medicação. Terapia diretamente observada.

\section{Treatment Recorded in Directly Observed Video: a possible tool in mental health}

\begin{abstract}
We will describe the experience of using Directly Observed Video Recorded Therapy with a mental health user in a city in the Recôncavo da Bahia. The videos were sent by Multimedia Messaging Service (MMS). This technique was decisive for her daily use of her medications without any failure, which enabled a rapid improvement of her delusional production. We suggest that the introduction of directly observed recordings in the acute period of severe mental disorders could favor a better therapeutic outcome, since it would provide the safety of the correct use of medications at this time.
\end{abstract}

Keywords: Mental health. Adhesion to medication. Directly observed therapy.

* Médico psiquiatra. Mestre em Medicina e Saúde Humana pela Escola Bahiana de Medicina e Saúde Pública. Professor assistente do Centro de Ciências da Saúde da Universidade Federal do Recôncavo da Bahia - UFRB.

** Graduando em Medicina da UFRB.

***Enfermeira e Doutora em Ciências pela EEUSP-SP e Professora assistente do Centro de Ciências da Saúde da UFRB.

****Psicóloga e Doutora em Ciências pela EEUSP-SP. Professora assistente do Centro de Ciências da Saúde da UFRB. 


\section{Introdução}

Portadores de transtornos mentais graves (TMG) podem necessitar de tratamento medicamentoso contínuo. O Ministério da Saúde do Brasil estima em 5 milhões de pessoas que precisam desta forma de cuidado (Brasil, 2011). No Transtorno Mental Grave - TMG, para um manejo medicamentoso efetivo, é preciso um tratamento continuado e de longa duração que envolve a redução ao máximo do número de recaídas, pois estas se relacionam com uma deterioração do quadro psíquico (Herz, Glazer, Mostert, Sheard, Szymanski, Hafez, Mirza \& Vana, 1991). Neste contexto, é preciso evidenciar que a não adesão a medicação gira em torno de $60 \%$, e é um significante fator de recaída e hospitalizações (Lieberman, Stroup, McEvoy, Swartz, Rosenheck, Perkins, Keefe, Davis, Davis, Lebowitz, Severe, \& Hsiao, 2005).

Pensando no contexto da longitudinalidade do cuidado, reflete-se que a não adesão ao tratamento medicamentoso é também, um grande problema para além de algumas situações de saúde mental. Como no caso de tuberculose, o qual tem chamado a atenção das autoridades sanitárias, o seu recrudescimento mundial, como no final dos anos 1990, em que um terço da população mundial estava infectada (WHO, 2016). Nesta perspectiva, a Organização Mundial de Saúde (OMS) propôs, então, como uma das principais estratégias de enfrentamento para o aumento dos casos de tuberculose, a técnica de Tratamento Diretamente Observado (TOD) (WHO, 2016).

Tal técnica consiste no uso das medicações específicas para a tuberculose diretamente observada por um profissional de saúde, e este evento pode ocorrer tanto na unidade de saúde quanto na residência ou, mesmo no local de trabalho do usuário em tratamento (WHO, 2016). O TOD aumenta a adesão terapêutica, diminui a recorrência da doença e previne o desenvolvimento de resistência às medicações (Wilkinson, 1994; Jereb, Simone e Onorato, 1999) . Existem algumas experiências no mundo com o Tratamento Gravado em Vídeo Diretamente Observado (Hoffman, Cunningham, Suleh, Sundsmo, Dekker, Vago, Munly, Igonya \& Hunt-Glassman, 2010). Esta técnica não visa a substituição do TOD, mas o seu uso em conjunto. $\mathrm{O}$ presente artigo teve como objetivo relatar a experiência de uma intervenção em uma Unidade Básica de Saúde, de um município do Recôncavo da Bahia, a fim de demostrar a utilização do Tratamento Gravado em Vídeo Diretamente Observado com uma usuária de saúde mental.

\section{História clínica, queixas e desenvolvimento:}

Relata-se o caso de Joana [nome fictício], 42 anos, possuía um filho, solteira, trabalhadora de nível médio na área da saúde, e encontrava-se afastada de suas atividades laborativas. Joana morava só, não possuía relacionamento amoroso e seus laços sociais eram restritos. Em 2011, foi afastada do trabalho por conta de alterações de pensamento que comprometiam seu juízo de realidade. Neste primeiro afastamento, referia que estava ocorrendo um complô entre suas colegas de trabalho para denegrir sua imagem, e o afastamento perdurou por três meses. Joana retornou ao trabalho por mais dois meses, porém a sensação de perseguição persistia e o convívio no trabalho foi deteriorando. Mais uma vez foi afastada de suas atividades laborativas, naquela ocasião por mais de um ano. Em ambos os afastamentos, Joana fazia uso irregular das medicações e, logo descontinuava o tratamento medicamentoso.

Em outra tentativa de retorno ao trabalho, Joana deixou a função que exercia em um serviço público de saúde, entretanto, o quadro persecutório persistia e ganhava contornos sexuais em que ela estava no centro da cena comprometendo sua eficiência e regularidade no serviço. Joana neste longo período não realizava acompanhamento psiquiátrico ou qualquer outra forma de cuidado com outros profissionais de saúde mental. Em avaliação médica, Joana se apresentava com produção delirante de cunho persecutório cujo conteúdo remetia a uma erotomania. Em nenhum momento, referia produção alucinatória de qualquer ordem. Refletiu-se na possibilidade de um diagnóstico de transtorno delirante persistente. Foi prescrito o antipsicótico risperidona na dosagem de 01 miligrama ao dia e, recomendou-se que ela se afastasse de suas atividades laborativas até melhora do quadro em questão.

Joana, ao longo dos meses, apresentava o seguinte padrão nas consultas: chegava francamente psicótica, mas aceitava fazer uso de algum tipo de terapêutica medicamentosa. Cumpriu o acordado em consulta por algumas semanas e, logo depois afastou-se do acompanhamento por meses. Foi observado este mesmo cenário por pelo menos três vezes. Este padrão de comportamento de Joana foi mantido até 30/01/2017, quando ela começou a relatar sobre ideação suicida. $\mathrm{Na}$ consulta, referia não ter mais sentido em viver e que se atirar de seu apartamento poderia ser uma saída. Joana mais uma vez apresentava produção delirante com conteúdo erotomaníaco, pensava, inclusive, em abandonar toda sua vida e ir para um país de língua inglesa, pois os homens do Brasil não estavam a deixando em paz, e ela já tinha trancado seu curso 
universitário e iniciado um curso intensivo de idioma estrangeiro. Como conduta medicamentosa, foi sugerido um antipsicótico de depósito e, também solicitado que ela fornecesse o contato de alguém próximo. Joana se recusou a fazer uso da medicação de depósito e propôs que todas as noites iria filmar o uso da sua medicação e enviar o vídeo. Em 01/02/2017 enviou o seu primeiro vídeo e, assim, seguiu diariamente. Os vídeos foram enviados por Serviço de Mensagens Multimídia (SMS). Na primeira avaliação, após os envios dos vídeos por 4 meses, Joana tinha retomado seu curso universitário e, não mais falava em mudar-se de país. O restante de seu exame psíquico estava dentro dos padrões esperados.

Esta técnica - tratamento gravado em vídeo diretamente observado - foi decisiva para um desfecho terapêutico positivo da usuária, pois Joana fez uso das medicações sem qualquer falha, o que possibilitou uma rápida melhora de sua produção psicótica. Foi possível, ainda, visualizar o ato de deglutição das medicações. No caso de Joana era 01 (um) comprimido de risperidona, 02 $\mathrm{mg}$ à noite. No período em questão, apenas por duas vezes não foi possível visualizar completamente a deglutição das medicações. Vale dizer que, os vídeos gravados por Joana eram enviados diretamente para o aparelho celular do médico assistente integrante da equipe do serviço de saúde e, posteriormente foram deletados. A usuária assinou um termo de consentimento livre e esclarecido para que sua experiência pudesse ser utilizada em um relato de caso, e publicação em um periódico científico, garantindo-lhe o anonimato.

Com o passar do tempo, a usuária não mais apresentava qualquer ideação suicida. Em uma demonstração de autocuidado, instalou em seu apartamento grades de proteção para que se as ideias suicidas mais uma vez ocorressem, ela estaria mais protegida. Ressaltamos que além do uso correto de suas medicações, foi possível observar os horários que ela as utilizava. Em consulta, Joana por vezes reclamava de uma sonolência no período da manhã, com os vídeos, ficou claro que na maioria das vezes a usuária fazia uso de suas medicações depois das 23 horas. Foi trabalhada com ela esta questão e, a queixa de sonolência diurna não mais aconteceu. Outro benefício que a técnica dos vídeos enviados foi o fortalecimento do vínculo entre a usuária e o profissional médico que era sua referência no serviço. Era notório o quanto por meio deles Joana se sentia mais acolhida, além de mostrar a utilização das medicações a mesma fazia pequenos comentários sobre seu dia. Em última entrevista, realizada em 01 de junho de 2017, Joana demonstrou o quanto ficou feliz com essa experiência e, revelou que nunca ficou por tanto tempo fazendo o uso correto de suas medicações. Relatou que, atualmente, entende a importância de suas medicações e que elas a deixam menos impulsiva, mais serena e afastam os pensamentos ruins.

\section{Discussão}

Em estudo, realizado no Quênia, os participantes que estavam sob tratamento da tuberculose, gravavam dos seus próprios aparelhos celulares, o uso das medicações e transmitiam por SMS para uma central (Hoffman et al, 2010). Foi observado uma melhora da adesão ao tratamento medicamentoso e, uma melhor relação custo- efetividade quando comparada a outras modalidades terapêuticas. Os participantes referiram um sentimento de ser especial e o profissional de saúde que participou do estudo, referiu uma sensação de estar provendo um serviço de saúde efetivo.

Outro estudo relacionado com saúde mental, tratava da utilização de vídeos diretamente observados durante a utilização de medicamentos em uma unidade de internamento. $\mathrm{O}$ objetivo era identificar aqueles pacientes que seriam, posteriormente ao internamento, os que apresentariam questões relacionadas a não adesão medicamentosa (Byrne, Deane, Murugesan \& Connaughton, 2014).

Partindo do pressuposto que a adesão terapêutica é um dos principais problemas relacionados a dificuldade do manejo de pessoas portadoras de TMG, pode-se pensar que a utilização da gravação em vídeo diretamente observada no manejo dos pacientes portadores de TMG poderá ser um caminho para um melhor desfecho terapêutico deste público. Cabe acrescentar que a utilização da gravação em vídeo diretamente observada garante a adesão de mais de $90 \%$ no correto uso das medicações (Jereb, Simone \& Onorato, 1999; DeMaio, Schwartz, Cooley \& Tice, 2001; Creary, Gladwin, Byrne, Hildesheim \& Krishnamurti, 2014; Chuck, Robinson, Macaraig, Alexander \& Burzynski, 2016).

\section{Conclusão}

Ressaltamos que apesar do caráter crônico dos transtornos mentais graves, estes podem apresentar episódios de agudização proporcionados na maioria das vezes por uma adesão terapêutica medicamentosa baixa. A introdução das gravações diretamente observadas no período agudo dos TMG poderia favorecer um melhor desfecho terapêutico no controle dos sintomas, pois traria a segurança do correto uso das medicações, nestes momentos. Pensando em um país de dimensões continentais como o Brasil, utilizar 
o SMS através de um aparelho celular reduz distâncias, podendo promover um melhor gerenciamento do tempo de trabalho das equipes de saúde e, individualizar o horário das gravações, de acordo com as necessidades particulares das pessoas em sofrimento psíquico.

\section{Referências:}

Byrne, M. K., Deane, F. P., Murugesan, G., \& Connaughton, E. (2014). Interrater reliability of the observer $\mathrm{R}$ acting of Medication Taking scale in an inpatient mental health facility. International journal of mental health nursing, 23(6), 498-505.

BRASIL. Ministério da Saúde. (2011). Saúde Mental no SUS: as novas fronteiras da Reforma Psiquiátrica. Relatório de Gestão 2007-2010.

Chuck, C., Robinson, E., Macaraig, M., Alexander, M., \& Burzynski, J. (2016). Enhancing management of tuberculosis treatment with video directly observed therapy in New York City. The International Journal of Tuberculosis and Lung Disease, 20(5), 588-593.

Creary, S. E., Gladwin, M. T., Byrne, M., Hildesheim, M., \& Krishnamurti, L. (2014). A pilot study of electronic directly observed therapy to improve hydroxyurea adherence in pediatric patients with sickle $\square$ cell disease. Pediatric blood \& cancer, 61(6), 1068-1073.

DeMaio, J., Schwartz, L., Cooley, P., \& Tice, A. (2001). The application of telemedicine technology to a directly observed therapy program for tuberculosis: a pilot project. Clinical infectious diseases, 33(12), 2082-2084.
Herz, M. I., Glazer, W. M., Mostert, M. A., Sheard, M. A., Szymanski, H. V., Hafez, H., Mirza, M. \& Vana, J. (1991). Intermittent vs maintenance medication in schizophrenia: two-year results. Archives of General Psychiatry, 48(4), 333-339.

Hoffman, J. A., Cunningham, J. R., Suleh, A. J., Sundsmo, A., Dekker, D., Vago, F., Munly K., Igonya E. K. \& Hunt-Glassman, J. (2010). Mobile direct observation treatment for tuberculosis patients: a technical feasibility pilot using mobile phones in Nairobi, Kenya. American journal of preventive medicine, 39(1), 78-80.

Jereb, J. A., Simone, P. M., \& Onorato, I. M. (1999). Directly observed therapy and tuberculosis treatment completion. American journal of public bealth, 89(4), 603-604

Lieberman, J. A., Stroup, T. S., McEvoy, J. P., Swartz, M. S., Rosenheck, R. A., Perkins, D. O., Keefe R. S. E., Davis S. M., Davis C. E., Lebowitz B. D., Severe J., Hsiao J. K. \& Severe, J. (2005). Effectiveness of antipsychotic drugs in patients with chronic schizophrenia. New England Journal of Medicine, 353(12), 1209-1223.

Wilkinson, D. (1994). High-compliance tuberculosis treatment programme in a rural community. The Lancet, 343(8898), 647-648.

World Health Organization. (2016). World tuberculosis report 2015. World Health Organization.

Submetido em: 28-7-2019

Aceito em: 17-12-2019 